\title{
Criopreservación de espermatozoides de anchoveta peruana (Engraulis ringens)
}

\author{
Cryopreservation of Peruvian anchovy (Engraulis ringens) sperm \\ Christian Catcoparco $^{1}$, Enrique Dupré ${ }^{2}$ y Carlos Espinoza ${ }^{1}$
}

\author{
${ }^{1}$ Laboratorio de Biología Experimental - Instituto del Mar del Perú. \\ Esq. Gamarra y Valle s/n, Chucuito Callao, Perú \\ ${ }^{2}$ Departamento de Biología Marina, Facultad de Ciencias del Mar, \\ Universidad Católica del Norte. Casilla 117, Coquimbo, Chile \\ cespinoza@imarpe.gob.pe
}

\begin{abstract}
The present work shows a practical and of cheap methodology for Engraulis ringens sperm cryopreservation, with the purpose to optimize its reproduction techniques in captivity. The investigation was divided in three stages. The first one consisted to evaluate toxicity of four cryoprotectants; dimethylsulfoxide ( $\mathrm{ME}_{2} \mathrm{SO}$ ), ethanol (ET), propylene glycol (PG), glycerol (GL) to three molars concentrations (0.5 M; 1.0 $\mathrm{M}$ and $1.5 \mathrm{M}$ ) on spermatic motility. Motility percentages of sperms incubated in $\mathrm{ME}_{2} \mathrm{SO}$ were significantly bigger than that observed in others three cryoprotectants. In the second stage, the optimal freezing rates were determinate, using $\mathrm{ME}_{2} \mathrm{SO}$ only. In this case, the biggest motility percentages were obtained with $1.5 \mathrm{M} \mathrm{ME}_{2} \mathrm{SO}$ with freezing rates of -20 to $-40^{\circ} \mathrm{C} \mathrm{min}^{-1}$ $(61.3 \pm 7.6 \%$ and $53.8 \pm 4.9 \%$ respectively). Finally, the effect of addition of not permeable cryoprotectant in cryoprotective solution (egg hen yolk, VHG) was evaluated to optimize the post-thawing spermatic motility; nevertheless, the results did not change significantly respect to control without VHG.
\end{abstract}

Key words: Sperm motility, cryoprotectans, $\mathrm{ME}_{2} \mathrm{SO}$, freezing rates
Resumen.- El presente trabajo muestra una metodología práctica y de bajo costo para criopreservar espermatozoides de Engraulis ringens, con la finalidad de optimizar las técnicas de su reproducción en cautiverio. La investigación fue dividida en tres etapas; la primera consistió en evaluar la toxicidad de cuatro crioprotectores; dimetilsulfóxido ( $\mathrm{ME}_{2} \mathrm{SO}$ ), etanol (ET), propilenglicol (PG) y glicerol (GL) a tres concentraciones molares diferentes $(0,5 \mathrm{M} ; 1,0 \mathrm{M}$ y 1,5 M) sobre la motilidad espermática. Los porcentajes de motilidad de espermatozoides incubados en $\mathrm{ME}_{2} \mathrm{SO}$ fueron significativamente mayores al de los observados con los otros tres crioprotectores. En la segunda etapa se determinó la tasa de congelamiento óptima, utilizando el crioprotector con el que se obtuvieron los mejores resultados de motilidad ( $\left.\mathrm{ME}_{2} \mathrm{SO}\right)$. En este caso, los mayores porcentajes de motilidad post-descongelamiento obtenidos fueron con $\mathrm{ME}_{2} \mathrm{SO}$ a $1,5 \mathrm{M}$ a tasas de congelamiento de -20 hasta $-40^{\circ} \mathrm{C}$ $\min ^{-1}(61,3 \pm 7,6 \%$ y 53,8 $\pm 4,9 \%$ respectivamente). Por último, se evaluó el efecto de la adición de un crioaditivo no permeable en la solución crioprotectora (vitelo de huevo de gallina, VHG), para optimizar la motilidad espermática post-descongelamiento; sin embargo, los resultados no variaron significativamente con los controles sin VHG.

Palabras clave: Motilidad espermática, crioprotectores, $\mathrm{ME}_{2} \mathrm{SO}$, tasas de congelamiento

\section{Introducción}

La reproducción de organismos marinos ocurre bajo ciertas condiciones ambientales, las cuales al variar generan alteraciones en los procesos de maduración gonadal, atresia ovocitaria, calidad y cantidad de desoves (Funamoto \& Auki 2002, Takasuka et al. 2005). La importancia del conocimiento de aquellos procesos en especies de importancia pesquera como la anchoveta peruana, Engraulis ringens Jenyns, 1842 (Espinoza et al. 2009), radica en las consecuencias que tienen sobre sus reclutamientos y posterior biomasa de los stocks. Para ello, también es necesario evaluar los efectos de la variabilidad de factores ambientales sobre la supervivencia y crecimiento de embriones y larvas, lo cual requiere un suministro constante de huevos en condiciones de cautiverio. Por tal motivo, fue necesario reproducir a esta especie en cautiverio mediante técnicas de inducción hormonal (Cisneros et al. 2006¹). No obstante, estas pruebas tuvieron el problema de asincronía

${ }^{1}$ Cisneros P, C Espinoza, B Buitrón, A Perea, V Vera, M Valdivia \& D Vizziano. 2006. Efecto de (GnRHa) y domperidona sobre el desove en cautiverio de la anchoveta peruana Engraulis ringens. XV Reunión Científica ICBAR, 7-9 agosto 2006, Universidad Nacional Mayor de San Marcos, Lima. Libro de Resúmenes, p. 86. 
entre el desove de hembras y la espermiación de machos, por lo que se propuso la criopreservación de espermatozoides como alternativa de solución para culminar con éxito la fecundación de ovocitos.

Para implementar las técnicas de criopreservación de espermatozoides a una especie es necesario evaluar y optimizar cada una de las etapas del proceso de congelamiento de células vivas, desde la incubación en los crioprotectores, hasta el congelamiento y descongelamiento. El presente estudio muestra una metodología práctica y de bajo costo, para criopreservar espermatozoides de Engraulis ringens, con la finalidad de optimizar las técnicas de su reproducción en cautiverio, evaluando el efecto de diferentes crioprotectores y tasas de congelamiento.

\section{Material y métodos}

\section{Obtención y acondicionamiento de los peces}

Ejemplares de Engraulis ringens, fueron capturados en la bahía del Callao, Perú, utilizando un sistema de red izada y luces de atracción según metodología descrita por Espinoza et al. (2008). El acondicionamiento de los peces al cautiverio se realizó durante 30 días en tanques cilíndricos de fibra de vidrio de $10 \mathrm{~m}^{3}$ de capacidad conectados a un sistema de recirculación de agua. Se mantuvo constante la temperatura a $16^{\circ} \mathrm{C}$ y fotoperiodo de 10L-14O; los peces fueron alimentados con LARVAL AP $100^{\circledR}\left(4,53 \mathrm{Kcal} \mathrm{g}^{-1}\right)$ y alimento extruido NICOVITA ${ }^{\circledR}$ $\left(4,61 \mathrm{Kcal} \mathrm{g}^{-1}\right)$ según metodología descrita por Espinoza et al. (2008).

\section{Inducción de espermiación y recolección de semen}

Grupos de 10 peces (hembras y machos con tallas mayores a $15 \mathrm{~cm}$ ) fueron anestesiados con $80 \mathrm{mg} \mathrm{L}^{-1}$ de MS-222 durante $3 \mathrm{~min}$, para luego inyectarles intraperitonealmente $0,01 \mu \mathrm{g}$ GnRHa g ${ }^{-1}$ de pez, según metodología descrita por Cisneros et al. (2006 $\left.{ }^{1}\right)$. Transcurridas 12 horas a $16^{\circ} \mathrm{C}$, se procedió a realizar una ligera presión abdominal a todos los peces inyectados para obtener muestras de semen de los machos. Se recolectaron muestras de $10 \mu \mathrm{L}$ de semen colocando una micropipeta automática directamente sobre el gonoporo para evitar la contaminación de la muestra con orina y/o heces lo cual hubiese afectado negativamente la motilidad espermática (Dreanno et al. 1998). La muestra se colocó en una cámara de cultivo celular conteniendo $90 \mu \mathrm{L}$ de solución salina al 0,9\%, para mantener inactivos a los espermatozoides durante el pre-congelamiento. Para obtener una concentración de espermatozoides adecuada para el conteo, como se describe mas adelante, se realizó una segunda dilución (1/40) en solución salina.

\section{Determinación de toxicidad de los crioprotectores}

Se evaluaron cuatro crioprotectores; dimetilsulfóxido ( $\mathrm{ME}_{2} \mathrm{SO}$ ), propilenglicol (PG), glicerol (GL) y etanol (ET) a tres concentraciones molares; 0,5 M; 1,0 M y 1,5 M. El periodo de incubación (tiempo de equilibrio) fue de $10 \mathrm{~min}$ a $8{ }^{\circ} \mathrm{C}$ para todos los casos. En un criotubo de $1,5 \mathrm{~mL}$ se mezclaron $5 \mu \mathrm{L}$ de semen inactivo con $195 \mu \mathrm{L}$ de crioprotector a la concentración requerida durante 10 min. Posteriormente la muestra fue diluida (1/20) en agua de mar para activar los espermatozoides y determinar el porcentaje de espermatozoides móviles (ver mas adelante) a los 0, 5 y 10 min post activación en agua de mar. Para cada experimento se realizaron nueve réplicas.

\section{Determinación de tasas óptimas de congelamiento en $\mathrm{ME}_{2} \mathrm{SO}$}

Muestras de semen de $10 \mu \mathrm{L}$ fueron diluidas $1 / 10$ en solución salina, para posteriormente obtener sub-muestras de $5 \mu \mathrm{L}$ que fueron distribuidas en criotubos conteniendo $195 \mu \mathrm{L}$ de $\mathrm{ME}_{2} \mathrm{SO}$ a $0,5 \mathrm{M} ; 1,0 \mathrm{M}$ ó 1,5 M. El congelamiento se realizó en un congelador mecánico portátil a una tasa constante hasta los $-100^{\circ} \mathrm{C}$, evaluándose cinco tasas de congelamiento $-10,-20,-30,-40$ ó $-50{ }^{\circ} \mathrm{C}$ $\min ^{-1}$, de acuerdo a la metodología descrita por Dupré \& Espinoza (2004). Después de 24 h, las muestras fueron descongeladas por inmersión de los criotubos en agua a $50^{\circ} \mathrm{C}$ durante $20 \mathrm{~s}$ e inmediatamente después en agua a temperatura ambiente hasta su descongelación total. Las muestras descongeladas fueron diluidas en agua de mar en una proporción de 1/20 y se calculó el porcentaje de espermatozoides móviles.

\section{Efecto del vitelo de huevo de gallina (VHG) como crioaditivo no permeable}

Para ello se elaboró una solución crioprotectora de $\mathrm{ME}_{2} \mathrm{SO}$ 1,5 M con VHG al 10\% (v/v) ( $\left.\mathrm{ME}_{2} \mathrm{SO}+\mathrm{VHG}\right)$, la cual se centrifugó a $1000 \mathrm{rpm}$ durante $10 \mathrm{~min}$ y se separó el sobrenadante para mantenerlo refrigerado a $5^{\circ} \mathrm{C}$ hasta su posterior uso. Como control se consideró la solución crioprotectora en base a $\mathrm{ME}_{2} \mathrm{SO}$ 1,5 M. El procedimiento de incubación en solución crioprotectora, congelamiento, descongelamiento y dilución en agua de mar para la posterior evaluación de la motilidad fue el mismo descrito anteriormente.

\section{Evaluación de la motilidad espermática}

Para determinar la motilidad espermática de las muestras sometidas a cada uno de los protocolos antes mencionados, se colocó una alícuota de la muestra diluida en agua de mar en un hematocitómetro y bajo microscopía óptica a 400 X se calculó el porcentaje de 
espermatozoides móviles a los 0, 5 y 10 min (Dupré \& Espinoza 2004).

\section{Análisis estadístico}

Se evaluó la normalidad de los datos utilizando la prueba Lilliefors y la homogeneidad de varianzas con la prueba de Cochrane. Para determinar diferencias significativas entre los porcentajes de motilidad espermática post incubación en diferentes crioprotectores se utilizó $t$ Student con corrección de Bonferroni. Los resultados de las pruebas de tasas de congelamiento fueron analizados mediante ANDEVA y la prueba de Tukey para las comparaciones entre tasas de congelamiento, mientras que $t$-Student con corrección de Bonferroni para las comparaciones entre diferentes concentraciones de crioprotector o diferentes soluciones crioprotectoras según fuese el caso. Para todas las pruebas se realizaron nueve réplicas y se consideró un nivel de significancia de 0,05. Se utilizó el programa SYSTAT 8.0 para Windows.

\section{Resultados}

\section{Motilidad espermática post-incubación en diferentes crioprotectores}

Posterior a la incubación en crioprotectores a concentraciones de $0,5 \mathrm{M}$; a los 0 min los valores de motilidad espermática variaron entre 60 y $90 \%$, observándose diferencias significativas sólo entre los resultados de $\mathrm{ME}_{2} \mathrm{SO}$ y GL $(90,0 \pm 4,9 \%$ y $66,2 \pm 3,8 \%$ respectivamente, $P<0,05)$. A los $5 \mathrm{~min}$, los mayores porcentajes de motilidad se obtuvieron con $\mathrm{PG}$ y $\mathrm{ME}_{2} \mathrm{SO}$ $(94,8 \pm 3,4 \%$ y $73,4 \pm 10,0 \%$ respectivamente) $\sin$ encontrarse diferencias significativas entre ambos valores $(P>0,05)$. A los $10 \mathrm{~min}$, los mayores porcentajes de motilidad fueron los obtenidos con PG $(96,4 \pm 15,8 \%)$ y $\mathrm{ME}_{2} \mathrm{SO}(54,1 \pm 13,8 \%)$, no encontrándose diferencias significativas entre ellos $(P<0,05)$ (Fig. 1$)$.

Cuando se utilizó cada uno de los crioprotectores a concentraciones de $1,0 \mathrm{M}$, el porcentaje de motilidad espermática a los 0 min post incubación en $\mathrm{ME}_{2} \mathrm{SO}$ (84,0 $\pm 4,7 \%$ ) fue significativamente diferente a los obtenidos con ET, GL y PG $(P<0,05)$. A los 5 y 10 min post incubación en $\mathrm{ME}_{2} \mathrm{SO}$, los valores de motilidad espermática se mantuvieron por encima del 50\% mientras que con los demás crioprotectores se obtuvieron valores menores al 40\% (Fig. 1).

A concentraciones de 1,5 M, los valores de motilidad espermática observados a los 0 y 5 min post incubación en $\mathrm{ME}_{2} \mathrm{SO}(70,3 \pm 4,3 \%$ y 42,9 $\pm 6,1 \%$ respectivamente) fueron significativamente diferentes a los obtenidos con

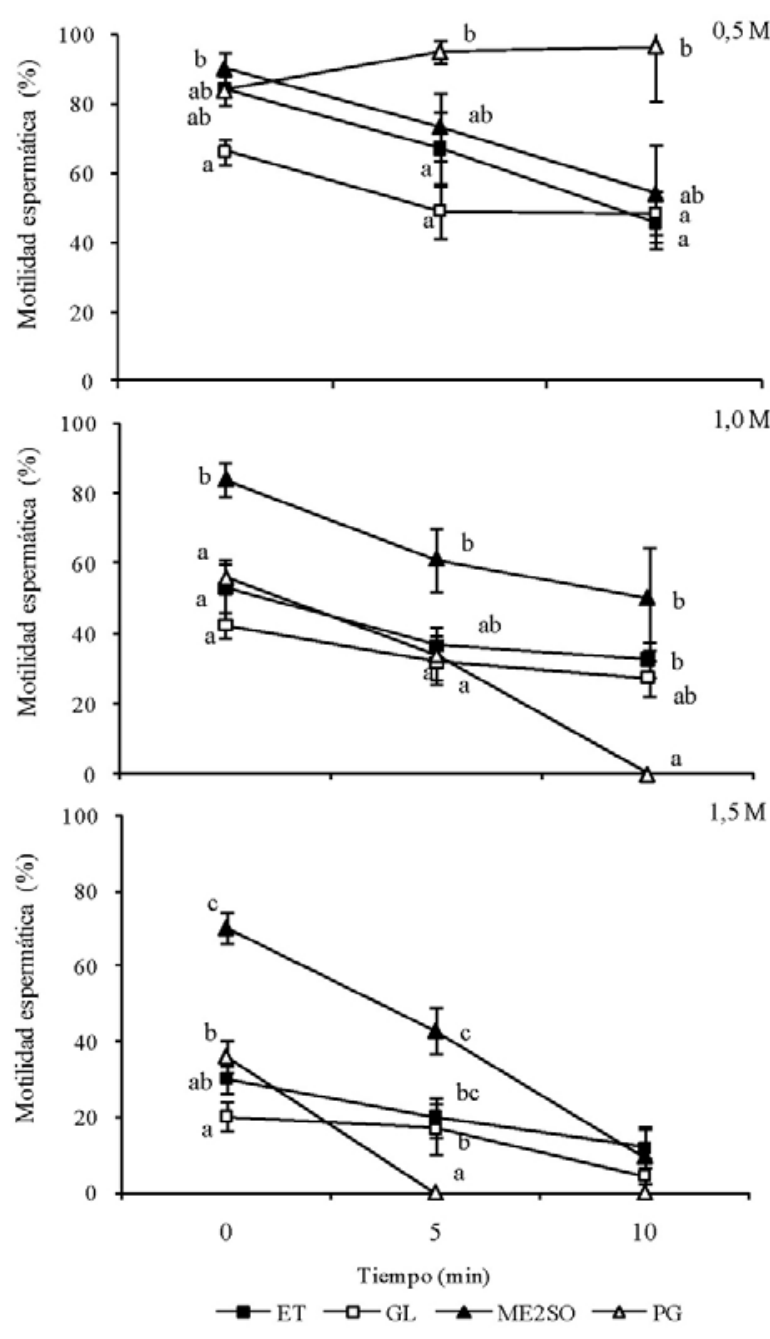

Figura 1

Motilidad total de espermatozoides de Engraulis ringens post-incubación en diferentes crioprotectores a diferentes concentraciones molares. ET: etanol,

GL: glicerol, $\mathrm{ME}_{2} \mathrm{SO}$ : dimetilsulfóxido,

PG: propilénglicol. Diferentes letras indican diferencias significativas entre tratamientos $(P<0,05)$

Total motility of Engraulis ringens spermatozoa postincubated in different cryoprotectants to different molar concentration. ET: ethanol, GL: glycerol, $\mathrm{ME}_{2} \mathrm{SO}$ : dimethyl sulfoxide, PG: propylene glycol. Different letters indicate significant differences between treatments $(P<0.05)$ 

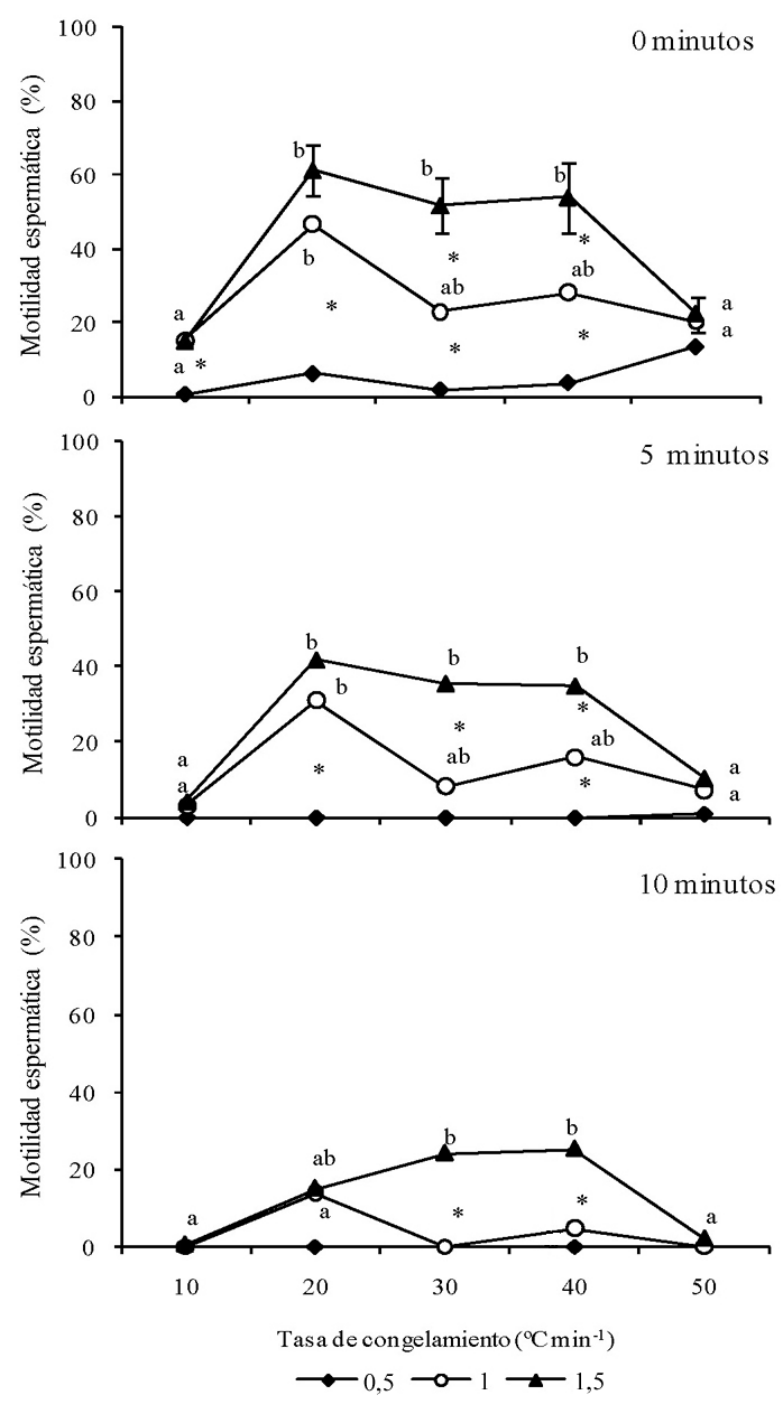

Figura 2

Motilidad total de espermatozoides congeladosdescongelados de Engraulis ringens utilizando $\mathrm{ME}_{2} \mathrm{SO}$ como agente crioprotector a diferentes concentraciones molares. Diferentes letras indican diferencias significativas entre tasas de congelamiento $y$ asteriscos indican diferencias significativas entre concentraciones molares $(P<0,05)$

Total motility of frozen-thawed spermatozoa of Engraulis ringens, using $\mathrm{ME}_{2} \mathrm{SO}$ as cryoprotectant to different molar concentrations. Different letters indicate significant differences between freezing rates and asterisks indicate significant differences between molar concentrations $(P<0.05)$
ET, GL y PG $(P<0,05)$. Los valores de motilidad espermática observados a los 10 min post incubación en los cuatro crioprotectores fueron menores al 20\%, no encontrándose diferencias significativas entre ellos $(P<$ 0,05) (Fig. 1).

\section{Determinación de tasas de congelamiento óptimas}

Estas pruebas se realizaron sólo con $\mathrm{ME}_{2} \mathrm{SO}$, que fue el crioprotector con el que se obtuvieron los mayores porcentajes de motilidad espermática en las pruebas de toxicidad. A los $0 \mathrm{~min}$, los mayores porcentajes de motilidad correspondieron a espermatozoides congelados a tasas de $-20,-30$ y $-40{ }^{\circ} \mathrm{C}$ min $^{-1}$ en $\mathrm{ME}_{2} \mathrm{SO} 1,5 \mathrm{M}(61,3$ $\pm 7,6 \% ; 51,7 \pm 9,7 \%$ y $53,8 \pm 4,9 \%$ respectivamente), valores significativamente diferentes a los obtenidos con tasas de -10 y $-50{ }^{\circ} \mathrm{C} \min ^{-1}(P<0,05)$. Además, los valores más altos fueron los obtenidos a concentraciones de 1,5 $\mathrm{M}$ respecto a los de 1,0 y 0,5 M $(P<0,05)$ (Fig. 2).

A los 5 min los valores de motilidad disminuyeron significativamente, manteniéndose la misma tendencia que a los 0 min (Fig. 2), mientras que a los 10 min los valores porcentuales de motilidad espermática no superaron el 25\% a excepción de los espermatozoides congelados en $\mathrm{ME}_{2} \mathrm{SO}$ a tasas de $-30 \mathrm{y}-40{ }^{\circ} \mathrm{C} \mathrm{min}^{-1}(24,2$ $\pm 7,1 \%$ y $25,3 \pm 10,0 \%$ respectivamente) (Fig. 2 ).

\section{Efecto de la adición de VHG como crioaditivo}

Los porcentajes de motilidad espermática observados a los 0 min post-dilución en agua de mar, de espermatozoides congelados en $\mathrm{ME}_{2} \mathrm{SO}+\mathrm{VHG}$ fueron mayores con la tasa de $-40^{\circ} \mathrm{C} \min ^{-1}(63,5 \pm 4,9 \%)$, valor significativamente diferente a los obtenidos con las tasas de congelamiento de $-10,-20 \mathrm{y}-50^{\circ} \mathrm{C} \mathrm{min}^{-1}$, con los cuales se obtuvieron valores menores al 15\% $(P<0,05)$ (Fig. 3).

Al comparar los valores de motilidad de espermatozoides congelados en $\mathrm{ME}_{2} \mathrm{SO}+\mathrm{VHG}$ con los congelados en $\mathrm{ME}_{2} \mathrm{SO}(32,9 \pm 7,6 \%$ y 61,3 $\pm 6,6 \%$ respectivamente), sólo se observaron diferencias significativas al congelar a tasas de $-20^{\circ} \mathrm{C} \min ^{-1}(P<0,05)$ (Fig. 3).

\section{Discusión}

Utilizando $\mathrm{ME}_{2} \mathrm{SO}$ en las tres concentraciones evaluadas se logró obtener porcentajes de motilidad de hasta $90 \%$, por lo cual sería el crioprotector menos tóxico para espermatozoides de E. ringens, respecto al ET, GL y PG. Por otro lado, a pesar que la incubación de espermatozoides en ET, GL y PG a bajas concentraciones permitió obtener porcentajes de motilidad espermática 


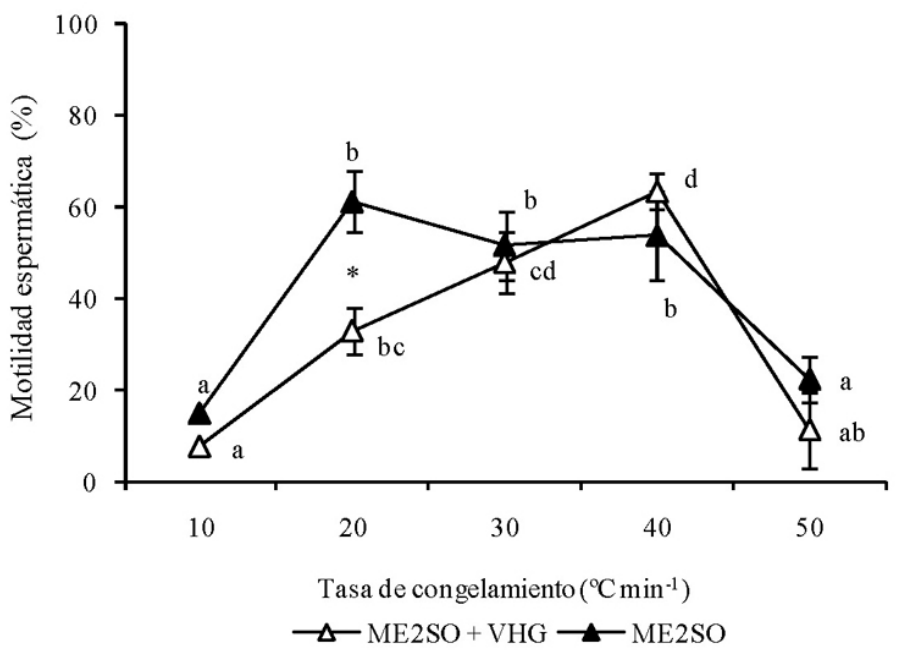

Figura 3

Motilidad total de espermatozoides congelados-descongelados de Engraulis ringens utilizando $\mathrm{ME}_{2} \mathrm{SO}+\mathrm{VHG}_{\text {ó }} \mathrm{ME} \mathrm{SO}_{2}$ VHG: vitelo de huevo de gallina. Diferentes letras indican diferencias significativas entre tasas de congelamiento y asteriscos indican diferencias significativas entre $\mathrm{ME}_{2} \mathrm{SO}+\mathrm{VHG}$ y $\mathrm{ME}_{2} \mathrm{SO}(P<0,05)$

Total motility of frozen-thawed spermatozoa of Engraulis ringens, using $\mathrm{ME}_{2} \mathrm{SO}+\mathrm{VHG}$ or $\mathrm{ME}_{2} \mathrm{SO}$ as cryoprotectant. VHG: egg hen yolk. Different letters indicate significant differences between freezing rates and asterisks indicate significant differences between $\mathrm{ME}_{2} \mathrm{SO}+\mathrm{VHG}$ and $\mathrm{ME}_{2} \mathrm{SO}(P<0.05)$

de hasta $80 \%$, a concentraciones mayores fueron significativamente bajas respecto al $\mathrm{ME}_{2} \mathrm{SO}$, lo cual no aseguraba una adecuada crioprotección durante el congelamiento. Por tal motivo, se utilizó el $\mathrm{ME}_{2} \mathrm{SO}$ como agente crioprotector para las pruebas de congelamiento, el cual resultó ser un eficiente crioprotector de espermatozoides de E. ringens tal como lo es para otras especies marinas como Scophthalmus maximus (Chen et al. 2004) y Paralichthys olivaceus (Zhang et al. 2003).

Se observó que mayores concentraciones de crioprotector tienen mayor efecto tóxico sobre los espermatozoides, ya que los porcentajes de motilidad disminuyeron con el incremento de la concentración molar en las pruebas de toxicidad (Fig. 1). Sin embargo, una mayor concentración de crioprotector asegura mayor protección contra los procesos de formación de hielo, como se muestra en los resultados de las pruebas de congelamiento en que los mayores porcentajes de motilidad fueron obtenidos a concentraciones molares de 1,5 M (Fig. 2). Esto está reportado también por otros autores, los que muestran que a pesar de la mayor toxicidad de crioprotectores a mayores concentraciones, éstas tienen mejor efecto protector contra el congelamiento (Gwo 1993, Gwo et al. 2005, Liu et al. 2006, Tian et al. 2008).
Considerando que durante el proceso de congelamiento, el crioprotector no permeable cumple funciones complementarias a las del crioprotector permeable, tal como disminuir el punto de fusión de la solución crioprotectora o servir como buffer osmótico (Rana 1995); se utilizó VHG para optimizar los resultados con $\mathrm{ME}_{2} \mathrm{SO}$ en E. ringens. Sin embargo, la motilidad no mejoró significativamente. Por el contrario, en otras especies como Perca flavescens (Ciereszko et al. 1993), Argopecten purpuratus (Dupré \& Espinoza 2004) y fundamentalmente en salmonidos (Baynes \& Scott 1987), la adición de VHG mejoró las motilidades postdescongelación. Resultados similares a los nuestros son reportados en especies como Clupea pallasi y $S$. maximus en una revisión hecha por Suquet et al. (2000), quienes señalan que el VHG no tiene una acción positiva sobre la crioprotección de espermatozoides de algunas especies; lo que indica un claro efecto especie-específico de los crioaditivos durante el congelamiento, y que dependería de la estructura de sus membranas plasmáticas y del modo en que las moléculas del crioaditivo interactúan con ellas. En ese sentido, no debería descartarse la posibilidad de evaluar el efecto de otros crioaditivos utilizados, tales como leche liofilizada (Sztein et al. 2001) o glucosa (Gwo et al. 2005), para optimizar los resultados en E. ringens. 
En conclusión, durante las pruebas de congelamiento en espermatozoides de E. ringens, se determinó, que los mayores porcentajes de motilidad se obtuvieron utilizando $\mathrm{ME}_{2} \mathrm{SO}$ 1,5 M y tasas de congelamiento de -20, -30 y $-40^{\circ} \mathrm{C} \min ^{-1}$ y que la adición de VHG no genera un incremento significativo de motilidad espermática en esta especie. Finalmente, considerando que las tasas de fecundación no siempre se correlacionan significativamente con la motilidad de los espermatozoides (Richardson et al. 1999), y que otros factores no relacionados con este parámetro pueden alterar la capacidad fecundante del espermatozoide; es necesario continuar las investigaciones para evaluar los protocolos de criopreservación desarrollados a través de las tasas de fecundación e incidencia de daños en ADN (expresados como malformaciones y/o mortalidad de embriones y larvas producidas), permitiendo en conjunto optimizar las técnicas.

\section{Agradecimientos}

El financiamiento de este estudio fue dado por el proyecto "Determinación experimental en ambientes controlados de los rangos de tolerancia de especies indicadoras a los cambios en las principales variables ambientales” del IMARPE - Perú y el de su publicación por el proyecto Fondef D05 I-10246 otorgado al Prof. E. Dupré de la Facultad de Ciencias del Mar, Universidad Católica del Norte, Chile.

\section{Literatura citada}

Baynes S \& A Scott. 1987. Cryopreservation of rainbow trout spermatozoa: the influence of sperm quality, egg quality and extender composition on post-thaw fertility. Aquaculture 66: 53-67.

Chen SL, XS Ji, GC Yu, YS Tian \& ZX Shang. 2004. Cryopreservation of sperm from turbot (Scophthalmus maximus) and application to large-scale fertilization. Aquaculture 236: 547-556.

Ciereszko A, L Ramseyer \& K Dabrowski. 1993. Cryopreservation of yellow perch semen. The Progressive Fish-Culturist 55: 261-264.

Dreanno C, M Suquet, E Destruyeres, J Cosson, H Le Delliou \& R Billard. 1998. Effect of urine on semen quality in turbot (Psetta maxima). Aquaculture 169: 247-262.

Dupré E \& C Espinoza. 2004. Congelamiento de espermatozoides del ostión del norte Argopecten purpuratus mediante congelador mecánico. Investigaciones Marinas 32: 3-9.
Espinoza C, A Perea, J Calderón, C Salazar, B Buitrón, V Vera, E Mecklenburg \& P Rojas. 2008. Captura y acondicionamiento en cautiverio de anchoveta peruana (Engraulis ringens). Informe Instituto del Mar del Perú 34: 269-277.

Espinoza C, V Vera, A Perea, B Buitrón, P Rojas \& O Kjesbu. 2009. Efecto de la ración alimenticia sobre la maduración gonadal y acumulación de grasa de anchoveta peruana (Engraulis ringens, Jenyns, 1842) en cautiverio. Latin American Journal of Aquatic Research 37: 181-190.

Funamoto T \& I Auki. 2002. Reproductive ecology of Japanese anchovy off the Pacific coast of eastern Honshu, Japan. Journal of Fish Biology 60: 154-169.

Gwo J. 1993. Cryopreservation of black grouper (Eninenhelus malabaricus) spermatozoa. Theriogenology 39: 1331-1342.

Gwo H, T Weng, L Fan \& Y Lee. 2005. Development of cryopreservation procedures for semen of Pacific bluefin tuna Thunnus orientalis. Aquaculture 249: 205-211.

Liu Q, J Li, S Zhang, F Ding, X Xu, Z Xiao \& S Xu. 2006. An efficient methodology for cryopreservation of spermatozoa of red seabream, Pagrus major, with 2-mL cryovials. Journal of the World Aquaculture Society 37: 289-297.

Rana K. 1995. Preservation of gametes. En: Bromage N \& R Roberts (eds). Broodstock management of egg and larval quality, pp. 53-73, Blackwell Scientific Publications, Oxford.

Richardson G, C Wilson, L Crim \& Z Yao. 1999. Cryopreservation of yellowtail flounder (Pleuronectes ferrugineus) semen in large straws. Aquaculture 174: 8994.

Suquet M, C Dreanno, C Fauvel, J Cosson \& R Billard. 2000. Cryopreservation of sperm in marine fish. Aquaculture Research 31: 231-243.

Sztein J, K Nobel, J Farley \& L Mobraaten. 2001. Comparison of permeating and nonpermeating cryoprotectants for mouse sperm cryopreservation. Cryobiology 41: 28-39.

Takasuka A, Y Oozeki, H Kubota, Y Tsuruta \& T Funamoto. 2005. Temperature impacts on reproductive parameters for Japanese anchovy: comparition between inshore and offshore waters. Fisheries Research 76: 475-482.

Tian YS, SL Chen, XS Ji, JM Zhai, LJ Sun, C Chen \& PZ Su. 2008. Cryopreservation of spotted halibut (Verasper variegatus) sperm. Aquaculture 284: 268-271.

Zhang YZ, SC Zhang, XZ Liu, YY Xu, CL Wang, MS Sawant, J Li \& SL Chen. 2003. Cryopreservation of flounder (Paralichthys olivaceus) sperm with a practical methodology. Theriogenology 60: 989-996. 